\title{
CANAL FORMAL E INFORMAL: ELECCIÓN DEL CONSUMIDOR
}

\author{
Melchor Cardona, Madeline ${ }^{1}$ \\ Universidad Autónoma de Occidente \\ mmelchor@uao.edu.co
}

Material original autorizado para su primera publicación en la revista académica REDMARKA. Revista Digital de Marketing Aplicado. https://doi.org/10.17979/redma.2016.01.016.4869

Recibido: 06 Mayo 2016

Aceptado: 17 Junio 2016

\section{Resumen}

La distribución de productos de consumo masivo realizada a través del canal formal (supermercados) e informal (tienda de barrio) permanece en pugna para sostenerse en un mercado altamente competitivo. La penetración en el mercado de estos canales difiere en Latinoamérica, USA y Europa por lo que se requiere de investigaciones que ayuden a precisar los motivos de preferencia de uno u otro canal. Los siguientes resultados aportan en la diferenciación de estos motivos encontrando que para el canal informal prima las relaciones sociales establecidas, la forma de entrega, disponibilidad del producto y valor agregado del servicio, mientras que para el canal formal cuenta las instalaciones, variedad, economía y comodidad. Los resultados pueden ser usados por representantes de canales de

\footnotetext{
${ }^{1}$ Magíster en Ingeniería Industrial, docente investigadora en Universidad Autónoma de Occidente. Coordinadora Grupo de Investigación en Mercadeo y Publicidad GIMPU.
}

REDMARKA UIMA-Universidad de A Coruña - CIECID 
distribución en la planeación de estrategias de mercadeo. Se hace un aporte en el método de análisis como una forma alternativa de precisar el análisis en variables comúnmente usadas en la investigación del mercado.

Palabras clave: Elección punto de venta, canal formal, canal informal, canales de distribución.

\section{Abstract}

Supermarkets and traditional grocery stores have played an important role in food and household merchandise distribution. It is a highly competitive market with different market distribution in Latin America, USA and Europe. In Latin America traditional store has stronger market presence mainly due to cultural factors, however, to enter more deeply to study this situation, we present the results of research conducted in the city of Santiago de Cali in Colombia in order to know the elements that consumer take into account after buying in Supermarkets or traditional stores. A quantitative study with multivariate statistical analysis allowed us to identify and differentiate these elements. Traditional channel is selected by social relationships established, delivery and value added service and availability. While for the formal channel is preferred because of the facilities, variety, economy and comfort.

Keywords: Consumer selection, formal channel, informal channel, distribution channels.

\section{Introducción}

REDMARKA UIMA-Universidad de A Coruña - CIECID 
El comercio y la distribución de productos de consumo masivo tienen como canales principales los supermercados y las tiendas localizadas en los barrios de las ciudades del país, los cuales se han sostenido como respuesta a las necesidades del consumidor, quien elige los canales por diferentes motivaciones. De acuerdo con el informe entregado por la Oficina Comercial de la Embajada de España en Colombia (2007) en los últimos años el comercio minorista ha experimentado un importante dinamismo a nivel mundial, mostrando una tendencia hacia la consolidación de holdings con presencia multinacional y a la expansión en el área de ventas y el fortalecimiento del multiformato. En el informe muestran que las grandes cadenas europeas como Carrefour, Ahold y Makro y las

norteamericanas como Wal Mart se encuentran posicionadas de manera estable y exitosa en los mercados latinoamericanos a través de alianzas estratégicas con supermercados locales o adquiriendo cadenas ya existentes. Las restricciones legales en Europa han incentivado esta expansión, la saturación de supermercados en muchos países europeos y la reducción de los costes de distribución que se logra con la interacción entre diferentes cadenas.

\section{Revisión de la literatura}

En Iglesias (2014) se muestra la distribución porcentual de la penetración del canal supermercados (formal) frente al canal tradicional (tienda de barrio) en algunos países: Chile: Formal $59 \%$ y el tradicional $41 \%$; en Brasil a $51 \%$ y $49 \%$, en Argentina a $40 \%$ y $60 \%$ y en Perú $25 \%$ y $75 \%$. La penetración de los supermercados en USA es $85 \%$ contra $15 \%$ de las tiendas; en Reino Unido es del $88 \%$ frente al $12 \%$ y en Francia los niveles son $81 \%$ y $19 \%$ respectivamente.

REDMARKA UIMA-Universidad de A Coruña - CIECID

Año IX, Número 16, (2016), v I pp. 5-27

http://www.redmarka.net/ ISSN 1852-2300 
Colombia ha tenido tradicionalmente un comercio minorista altamente competitivo, según el periódico El Tiempo (2014) el avance de los supermercados e hipermercados, se encuentra en el 53\% para el año 2014, mientras que el formal está en el $47 \%$. De acuerdo con el informe la gran cantidad de tiendas es consistente con el alto nivel de empleo informal y los bajos niveles de ingresos. En contraste con la elevada fragmentación del sector informal, se presenta una alta concentración en el sector formal, con la existencia de pocos participantes, la mayoría de las grandes cadenas minoristas regionales y globales que compiten con pocos o ningún minoristas locales formales.

Es por este posicionamiento del canal informal en el mercado, que los grandes supermercados han desarrollado diferentes estrategias de expansión buscando acercarse a los barrios por ejemplo con los formatos de proximidad (Iglesias, 2014) que consiste en almacenes de autoservicios más pequeños o tiendas express, también mediante alianzas con las tiendas para proveerlas tanto de marcas nacionales como de marcas propias, sin embargo, con relación a la permanencia de las tiendas en el mercado permanecerán aquellas que se sigan formalizando. Los gobiernos de los países de la región se encuentran en la búsqueda de recaudar más impuestos para financiar sus actividades, por lo tanto los pequeños negocios deben organizar la contabilidad y saber qué ingresos y gastos se tiene.

Adicionalmente, manifestó que las tiendas tienen retos que enfrentar a partir de la incursión de las tiendas express en los barrios, el mayor uso de las tarjetas de crédito y el comercio electrónico.

Bajo este contexto, la tienda de barrio sigue siendo un tema de especial interés para profundizar en el conocimiento de su dinámica y tendencia frente a su

REDMARKA UIMA-Universidad de A Coruña - CIECID 
sostenimiento en el mercado. Es por esta razón que ha sido objeto de investigaciones desde la economía, sociología, administración y el marketing. Unos se han dedicado a describir las características del mercado, y otros a identificar las razones sociales, culturales y personales por las cuales el canal tradicional permanece en un mercado altamente competitivo.

En la revisión de la literatura, se identificaron estudios concentrados en medir el impacto de las grandes superficies en el comercio minorista de los países estudiados; mientras que en otros estudios se incluyeron variables relacionadas con las características individuales del consumidor. Entre las primeras se encuentra: Gorton et al. (2011) en Tailandia y otros países de Asia oriental, Figuié y Moustier (2009) en Vietnam, Hino (2010) en Israel.

Se observa que en las investigaciones que se concentraron en identificar las características individuales de los consumidores, coinciden que los factores relevantes para realizar las compras en un supermercado o hipermercado son la disponibilidad de bienes en el momento que se requieren (Farhangmehr, Marques y Silva, 2000) mientras que la elección de compra en la tienda tradicional depende de la distancia a recorrer y la tendencia a encontrar productos perecederos (Goldman y Hino, 2005). Gary y Peter (2011) examinaron las actitudes y creencias de los compradores de productos comestibles en el contexto minorista australiano, encontrando que los factores de elección más importantes para los hombres son la velocidad, comodidad y eficiencia; mientras que para las mujeres son los precios, limpieza y calidad.

En los estudios realizados en América Latina, se identificaron, además de las anteriores, variables relacionadas con las relaciones sociales que se tejen en el

REDMARKA UIMA-Universidad de A Coruña - CIECID 
contexto de las tiendas como Díaz, Lacayo y Salcedo (2007) y en Colombia, Páramo y Ramírez (2009), Córdoba y Cano (2009).

Aunque en la revisión de la literatura se encontraron varias investigaciones internacionales sobre determinantes de elección de un punto de venta, son menos los trabajos realizados en Colombia; en especial los trabajos donde se identifican y comparan los determinantes de elección en tiendas y supermercados.

A partir de los hallazgos de los estudios anteriores y de la revisión de algunos modelos teóricos sobre elección de punto de venta del consumidor, se desarrolló la presente investigación buscando integrar los factores del entorno, del individuo y los determinantes del establecimiento que el consumidor tiene en cuenta al momento de elegir el supermercado y tiendas de barrio para sus compras, con el fin de entregar información confiable y contextualizada a la dinámica regional.

Los resultados y el método desarrollado puede ser fuente de consulta para los investigadores interesados en profundizar en el tema, dado que se podrá tomar como referente el diseño del marco conceptual, el enfoque integrado utilizado y el instrumento de medición.

Los resultados son de importancia para propietarios de tiendas y supermercados porque se entrega información precisa que servirá para tomar decisiones mejor respaldadas al momento de elegir sus estrategias de mercadeo y de servicio al cliente, en tanto conocerán que es lo que realmente motiva a los consumidores, la compra en tiendas y supermercados.

\section{Metodología}

REDMARKA UIMA-Universidad de A Coruña - CIECID 
Se desarrolló una investigación de tipo cuantitativa transversal simple, utilizando un cuestionario semiestructurado con cuarenta y nueve preguntas organizadas en cinco componentes: hábitos de compra en supermercados, hábitos de compra en tiendas, características de atención en la tienda y perfil de los encuestados. El cuestionario se aplicó de forma dirigida, en los hogares seleccionados en la muestra y a la persona encargada de hacer las compras del mercado en el hogar. Adicionalmente se hizo una pregunta filtro acerca de si realizaban compras en ambos establecimientos tiendas y supermercados. El trabajo de campo se realizó en el mes de abril del año 2013.

La población objeto de estudio se definió como los compradores de productos de la canasta familiar en tiendas y supermercados, de ambos sexos, mayores de 25 años, pertenecientes a los estratos 3,4 y 5 de la ciudad de Cali. En Colombia existe la distribución de la población de acuerdo con el estrato socioeconómico, la cual se hace con base en las características de las viviendas y su entorno. Se fundamenta principalmente y de acuerdo con el Departamento Administrativo Nacional de Estadística (DANE) para cobrar de manera diferencial los servicios públicos domiciliarios permitiendo asignar subsidios y cobrar contribuciones en esta área. De esta manera, quienes tienen más capacidad económica se encuentran en los estratos socioeconómicos más altos y pagan más por los servicios públicos y contribuyen para que los estratos bajos puedan pagar sus facturas. El tipo de muestreo utilizado fue aleatorio simple, trabajando con una muestra de 600 que corresponde a un error del $4 \%$ y una confianza del $95 \%$.

Para el análisis de la información, se utilizaron técnicas de análisis multivariado usando el método de Análisis de Componentes Principales (ACP) que tiene como principal objetivo la síntesis de la información o reducción de la dimensionalidad en número de variables. Es decir, a partir de un número de variables es posible

REDMARKA UIMA-Universidad de A Coruña - CIECID 
obtener un número reducido de componentes conformados por variables pero con la menor pérdida de información posible.

Este método multivariante se aplica a variables numéricas que tienen una relación lineal entre ellas. Sin embargo, para el caso de variables categóricas (nominales u ordinales) y sin el supuesto de relación lineal entre ellas, se cuenta con una variación del método llamado Análisis de Componentes Principales Categórico Categorical Principal Components Analysis (Catpca) (Linting et al., 2007).

De acuerdo con Molina y Espinosa (2010), es un método de escalamiento óptimo perteneciente a las técnicas no lineales de análisis multivariante, similar al ACP estándar, pues se utiliza para el mismo objetivo, pero a diferencia de éste, el Catpca es un método que permite escalar las variables a diferentes niveles de medida, permitiendo relaciones no lineales entre las mismas. Por tanto, el Catpca tiene la misma finalidad que el ACP estándar, pero aplicado a transformaciones no lineales de los datos (Molina y Espinosa, 2010).

Cuando se pretende sintetizar la información de un conjunto de variables, el ACP en el caso de variables cuantitativas y el Catpca cuando se opera con variables categóricas, son herramientas de gran utilidad. El procedimiento que utilizan ambos métodos para la extracción de factores es exactamente el mismo: la primera componente es aquella que maximiza el porcentaje de varianza explicada, por lo que la mayoría de las variables tienen una carga importante en esta dimensión. La segunda y siguientes componentes explican porcentajes cada vez más reducidos de varianza y suelen ser muy pocas las variables que presentan cargas elevadas. Esto hace que a la hora de interpretar las componentes, la primera de ellas sea la más relevante $\mathrm{y}$, a menudo, un factor resumen del concepto que se está estudiando. La segunda y siguientes dimensiones suelen ser

REDMARKA UIMA-Universidad de A Coruña - CIECID 
matices del fenómeno analizado con una importancia relativamente baja si se comparan con la primera. Esta solución es ideal cuando el objetivo que se persigue es explicar la mayor cantidad posible de información con el menor número de componentes.

El proceso tiene varias etapas. La primera es seleccionar las variables que explican el fenómeno en estudio y con ellas se realiza un análisis de componentes principales categórico con el algoritmo Catpca. Este algoritmo realiza dos procesos simultáneos: el escalamiento óptimo de las variables y la reducción de la dimensionalidad de las variables transformadas que se obtienen. El escalamiento es un método que atribuye valores numéricos óptimos a las diferentes categorías de respuesta. Estos valores numéricos son calculados por aproximaciones sucesivas con un procedimiento de iteración, a fin de optimizar la solución final. Como resultado, se obtiene un conjunto de variables que son transformaciones de las originales, pero a diferencia de aquellas, con propiedades métricas (Linting et al., 2007).

En algunos casos, no solo es necesaria la reducción de dimensionalidad, además se requiere descomponer y analizar un concepto general en un conjunto de dimensiones o factores que midan los diversos aspectos de los que se compone ese fenómeno. Por ejemplo, cuando se pretende analizar los factores que influyen en un determinado comportamiento o actitud, situación que se relaciona con el objeto de estudio de la presente investigación. En estos casos, es importante y necesario trabajar con una variación adicional del ACP que se refiere a la rotación de los factores. Este análisis adicional favorece una solución más simple y sencilla de interpretar con la posibilidad de no alterar las propiedades matemáticas de la estructura factorial.

REDMARKA UIMA-Universidad de A Coruña - CIECID 
Existen varios procedimientos parar realizar la rotación factorial y todos tienen en común la búsqueda de una estructura simple y, para todos el porcentaje de varianza total explicada no cambia, aunque sí el porcentaje de varianza explicado por cada uno de los factores. La rotación de factores ha sido desarrollada para el ACP, sin embargo Molina y Espinosa (2010) muestran en su artículo una solución para hacer la rotación en el Catpca.

Los autores muestran como una posibilidad para su desarrollo el guardar las variables transformadas que presentan cuantificaciones que son estimaciones numéricas "óptimas" de las categorías de las variables originales, lo que significa que los resultados que se obtendrían al realizar un nuevo análisis factorial clásico con estas variables serían prácticamente los mismos. De aquí se parte para obtener la rotación factorial, realizando un nuevo análisis factorial clásico a través del ACP (para variables numéricas) y utilizando lo que la rotación de los ejes que el procedimiento ofrece, entre ellas la VARIMAX que es un método de rotación ortogonal que minimiza el número de variables con cargas elevadas en un factor.

De acuerdo con los autores, la solución no rotada que se obtiene al aplicar el análisis factorial clásico a las variables transformadas coincide con la proporcionada por el algoritmo Catpca, pero a diferencia de éste, el primero facilita una solución rotada, simplificando la interpretación de las dimensiones subyacentes.

Las transformaciones dependen de la escala de medición de la variable y del número de dimensiones consideradas. En la presente investigación las variables utilizadas son nominales. Con relación a la cantidad de los factores que se van a considerar se aplicó el criterio de Kaiser-Guttman, mediante el cual se retienen aquellas dimensiones cuyo autovalor es mayor que la unidad. Esto implica que

REDMARKA UIMA-Universidad de A Coruña - CIECID 
cada factor explica un porcentaje de varianza mayor al explicado por cada variable original por sí misma.

Para el análisis de resultados se observa finalmente la matriz de componentes rotados, en la cual se identifica las variables con mayor peso o carga factorial para cada uno de los componentes. De esta manera se seleccionan las variables que forman parte de cada componente y de acuerdo con sus características se procede a darle a un nombre que refleje lo que la componente principal agrupa. Al final se obtiene entonces, un grupo pequeño de componentes que integran la totalidad de variables analizadas.

\section{Resultados}

Para identificar los elementos que el consumidor tiene en cuenta cuando va a comprar en la tienda y en el supermercado se aplicó el análisis de componentes principales para cada uno de los lugares a elegir: tiendas y supermercados. Se inició seleccionando las variables que se midieron para cada uno de los sitios de compra y se realizó el análisis correspondiente para cada caso.

Tiendas: Respecto a los criterios que tienen en cuenta las personas entrevistadas al momento de elegir a qué tienda va a ir son: me fían; es un lugar de confianza; el trato es respetuoso; el tendero es mi amigo/conocido; la atención es amable y personalizada; me encuentro/converso con amigos/vecinos; conocen mis gustos y preferencias; atienden rápido; venden presentaciones pequeñas; se puede comprar por unidades/poca cantidad; es económico/precios bajos; tiene amplios horarios (mañana y noche); el lugar es agradable; hay domicilios; los productos son frescos; es rápido ir y volver; hay variedad de productos/se consigue de todo; me sacan de apuros/se hacen compras de urgencia; queda cerca; los productos son de buena calidad.

REDMARKA UIMA-Universidad de A Coruña - CIECID

Año IX, Número 16, (2016), v I pp. 5-27

http://www.redmarka.net/ ISSN 1852-2300 
Para la reducción dimensionalidad variables que influyen en la compra en tiendas se ejecutó el procedimiento Catpca sobre las variables identificadas solicitando la agrupación sobre cuatro factores que explican el $32 \%$, como se observa en la tabla 1.

Tabla 1. Varianza explicada tiendas

\begin{tabular}{lrrr}
\hline \multirow{2}{*}{ Dimensión } & \multicolumn{2}{c}{$\begin{array}{c}\text { Alfa de } \\
\text { Cronbach }\end{array}$} & \multicolumn{2}{c}{ Varianza explicada } \\
\cline { 2 - 4 } & \multicolumn{1}{c}{$\begin{array}{c}\text { Total } \\
\text { (Autovalores) }\end{array}$} & $\begin{array}{c}\text { \% de la } \\
\text { varianza }\end{array}$ & \multicolumn{2}{c}{$\begin{array}{c}\text { Total } \\
\text { (Autovalores) }\end{array}$} \\
\hline 1 & 0,593 & 2291 & 11453 \\
\hline 2 & 0,357 & 1513 & 7565 \\
\hline 3 & 0,314 & 1425 & 7123 \\
\hline 4 & 0,238 & 1292 & 6462 \\
\hline Total & $0,891(\mathrm{a})$ & 6521 & 32604 \\
\hline
\end{tabular}

Fuente: Elaboración propia

Durante la ejecución del Catpca se solicita el guardar en la base de datos los valores numéricos estimados (cuantificaciones) para las categorías de cada variable, para luego proceder a aplicar el análisis factorial clásico con rotación VARIMAX, dando como resultado la matriz de cargas factoriales rotadas. Para la interpretación de la matriz factorial, se identifica las variables que tienen mayor peso en cada factor $y$ valores bajos en el resto y se eliminan las variables con cargas factoriales bajas dado que no muestran relación con ninguno de los factores (Hair et al., 1999). En la tabla 2 se muestra los componentes rotados.

Tabla 1. De componentes rotados tiendas 


\section{Entrega}

$\begin{array}{lccc}\text { Relacio } & \text { del } & \text { Valor } & \text { Disponi } \\ \text { nes } & \text { servicio } & \text { agrega } & \text { bilidad } \\ & y & \text { do } & \\ & \text { product } & & \end{array}$

0

\begin{tabular}{lrrrr}
\hline & 1 & 2 & 3 & 4 \\
\hline Me fían & 0,329 & 0,323 & $-0,433$ & $-0,045$ \\
\hline Es un lugar de confianza & 0,434 & $-0,173$ & 0,359 & $-0,018$ \\
\hline El trato es respetuoso & 0,618 & 0,055 & $-0,014$ & $-0,038$ \\
\hline El tendero es mi amigo/conocido & 0,490 & 0,102 & 0,242 & $-0,160$ \\
\hline La atención es amable y & 0,564 & $-0,070$ & 0,128 & $-0,055$ \\
personalizada & & & & \\
\hline Me encuentro/converso con & 0,650 & $-0,082$ & $-0,007$ & $-0,028$ \\
amigos/vecinos & & & & \\
\hline Conocen mis gustos y preferencias & 0,502 & 0,050 & $-0,347$ & 0,171 \\
\hline Atienden rápido & 0,065 & 0,091 & $-0,039$ & $-0,564$ \\
\hline Venden presentaciones pequeñas & 0,038 & 0,684 & $-0,074$ & 0,024 \\
\hline Se puede comprar por & $-0,142$ & 0,609 & 0,177 & 0,269 \\
unidades/poca cantidad & & & & \\
\hline Es económico/precios bajos & $-0,098$ & 0,128 & 0,162 & 0,078 \\
\hline Tiene amplios horarios (mañana y & 0,144 & $-0,071$ & 0,433 & $-0,048$ \\
noche) & & & & \\
\hline El lugar es agradable & 0,169 & 0,094 & 0,453 & $-0,213$ \\
\hline Hay domicilios & $-0,023$ & $-0,125$ & 0,223 & $-0,455$ \\
\hline Los productos son frescos & $-0,037$ & 0,179 & 0,473 & 0,252 \\
\hline Hay variedad de productos/se & 0,474 & $-0,067$ & $-0,068$ & 0,376 \\
\hline
\end{tabular}


CANAL FORMAL E INFORMAL:

ELECCIÓN DEL CONSUMIDOR

Melchor Cardona, Madeline

consigue de todo

\begin{tabular}{lllll}
\hline $\begin{array}{l}\text { Me sacan de apuros/se hacen } \\
\text { compras de urgencia }\end{array}$ & $-0,065$ & $-0,469$ & 0,043 & 0,280 \\
\hline Los productos son de buena calidad & 0,134 & $-0,085$ & 0,336 & 0,458 \\
\hline
\end{tabular}

Fuente: Elaboración propia

La primera dimensión presenta cargas elevadas en todas aquellas variables que miden la relación de confianza que se puede establecer en la tienda como la posibilidad de fiar, el recibir una atención respetuosa, el que conozcan los gustos y preferencias de los cliente y las variables que miden las relaciones que se pueden establecer con el tendero y con los vecinos. Por esta razón este componente se le asigna el nombre de relaciones.

En el segundo factor, las variables con mayor carga son aquellas que recogen la forma como se entrega el servicio y el producto en tanto la atención es rápida y se pueden hacer compras por unidades y/o en poca cantidad. Este componente se puede considerar como el que mide la entrega del servicio y el producto en la tienda.

Con respecto a la tercera dimensión, son las variables economía, horario, domicilios y alimentos frescos las que presentan mayores cargas. Estas variables miden el valor agregado de tener un establecimiento cerca y cubre los elementos importantes para el consumidor en tanto consigue precios económicos en horario amplio y además puede tenerlo a domicilio.

Las variables relacionadas con aspectos como variedad de productos y la posibilidad de encontrar de todo, el hacer compras de urgencia y calidad de los productos se agrupan en el cuarto componente. Estas características que el

REDMARKA UIMA-Universidad de A Coruña - CIECID 
consumidor resalta en la compra en tiendas, se pueden interpretar como la disponibilidad con la que cuenta la tienda sobre los productos que ofrece.

De esta manera se resumen en cuatro los aspectos considerados por los consumidores en las valoraciones que hacen al momento de decidir su compra en la tienda: las relaciones de confianza que se establecen, la forma en que reciben el servicio y los productos, el valor agregado y la disponibilidad.

Supermercado: Se midieron las siguientes variables para hacer el análisis en el supermercado: que quede cerca; por tradición; posibilidad de escoger los productos; se puede hacer un mercado grande; es amplio; los productos son frescos; hay variedad de sitios de comida; es vigilado/hay seguridad; buena higiene de los productos; colocan sillas en las cajas registradoras; hay parqueadero; hay buen orden y exhibición de productos; hay variedad de productos; hay variedad de marcas; hay variedad de precios; hay variedad de tamaños; los precios son económicos; los productos están en excelente estado; el personal es atento y dispuesto a resolver inquietudes; se encuentran productos nuevos; se puede cambiar el producto si sale en mal estado; los productos tienen adecuada fecha de vencimiento; se puede conseguir todo en un mismo sitio sea limpio; hay ofertas/promociones.

Se ejecutó el procedimiento Catpca sobre las variables identificadas solicitando la agrupación sobre cuatro factores que explican el $24 \%$ (ver tabla 3 )

Tabla 3. Varianza explicada supermercados

\begin{tabular}{lll}
\hline Dimensió & Alfa de & Varianza explicada \\
$\mathbf{n}$ & Cronbach & \\
\hline
\end{tabular}

REDMARKA UIMA-Universidad de A Coruña - CIECID 


\begin{tabular}{lrrr}
\hline & $\begin{array}{c}\text { Total } \\
\text { (Autovalo } \\
\text { res) }\end{array}$ & $\begin{array}{c}\text { \% de la } \\
\text { varianza }\end{array}$ & $\begin{array}{c}\text { Total } \\
\text { (Autovalore } \\
\text { s) }\end{array}$ \\
\hline 1 & 0,459 & 1788 & 7150 \\
\hline 2 & 0,435 & 1718 & 6874 \\
\hline 3 & 0,319 & 1442 & 5769 \\
\hline 4 & 0,229 & 1282 & 5128 \\
\hline Total & $0,874(a)$ & 6230 & 24921 \\
\hline
\end{tabular}

Fuente: Elaboración propia

Sobre las variables cuantificadas que el Catpca genera, se aplicó el análisis factorial clásico con rotación VARIMAX. La solución rotada que proporciona el análisis factorial aplicado a las variables transformadas facilita la interpretación de las dimensiones utilizadas las cuales se deben interpretar. La matriz de componentes rotados que se muestra en la Tabla 4.

Tabla 4. De componentes rotados supermercados

\begin{tabular}{lrrrrr}
\hline Componente & $\begin{array}{c}\text { Característ } \\
\text { icas físicas } \\
\text { del } \\
\text { supermerc } \\
\text { ado }\end{array}$ & $\begin{array}{c}\text { Varied } \\
\text { ad }\end{array}$ & $\begin{array}{c}\text { Calidad } \\
\text { Econo } \\
\text { mía }\end{array}$ & $\begin{array}{c}\text { Comodid } \\
\text { ad }\end{array}$ \\
& 1 & 2 & 3 & 4 \\
\hline Por tradición & 0,423 & 0,066 & $-0,064$ & $-0,248$ \\
\hline Es amplio & 0,553 & $-0,028$ & $-0,103$ & $-0,080$ \\
\hline Hay variedad de sitios de comida & 0,374 & $-0,020$ & $-0,133$ & 0,057 \\
\hline Es vigilado/hay seguridad & 0,277 & $-0,135$ & 0,080 & 0,216 \\
\hline Buena higiene de los productos & 0,507 & $-0,176$ & 0,302 & 0,045 \\
\hline
\end{tabular}

REDMARKA UIMA-Universidad de A Coruña - CIECID

Año IX, Número 16, (2016), v I pp. 5-27

http://www.redmarka.net/ ISSN 1852-2300 
CANAL FORMAL E INFORMAL:

ELECCIÓN DEL CONSUMIDOR

Melchor Cardona, Madeline

\begin{tabular}{lcccc}
\hline Hay parqueadero & 0,263 & 0,164 & $-0,142$ & 0,235 \\
\hline $\begin{array}{l}\text { Hay buen orden y exhibición de } \\
\text { productos }\end{array}$ & 0,369 & 0,018 & 0,220 & 0,126 \\
\hline Hay variedad de productos & 0,102 & 0,629 & 0,150 & 0,103 \\
\hline Hay variedad de marcas & $-0,087$ & 0,700 & 0,047 & 0,053 \\
\hline Hay variedad de precios & $-0,026$ & 0,597 & 0,070 & $-0,187$ \\
\hline Los precios son económicos & $-0,447$ & 0,034 & 0,364 & $-0,085$ \\
\hline Los productos están en excelente & $-0,092$ & $-0,062$ & 0,456 & $-0,079$ \\
estado & & & & \\
\hline $\begin{array}{l}\text { El personal es atento y dispuesto a } \\
\text { resolver inquietudes }\end{array}$ & 0,168 & $-0,064$ & 0,425 & 0,344 \\
\hline $\begin{array}{l}\text { Los productos tienen adecuada } \\
\text { fecha de vencimiento }\end{array}$ & 0,000 & 0,065 & 0,261 & 0,422 \\
\hline Sea limpio & & & & \\
\hline Hay ofertas/promociones & 0,093 & 0,139 & 0,109 & 0,437 \\
\hline
\end{tabular}

Fuente: Elaboración propia

En el primer factor, las variables relacionadas con las características físicas de los supermercados son las que se agrupan, tales como tradición, amplitud, variedad en sitios para comer, seguridad, higiene, parqueadero, orden y exhibición de productos.

En el segundo factor se observa con mayor carga aquellas variables que agrupan la variedad que se pueden encontrar en un supermercado frente a productos, marcas y precios.

REDMARKA UIMA-Universidad de A Coruña - CIECID 
La tercera dimensión presenta cargas elevadas en variables que miden la calidad y economía en los productos que ofrece el supermercado y en la atención en el servicio.

Finalmente, el cuarto componente agrupa variables relacionadas con los servicios que ofrecen comodidad, el manejo de la fecha de vencimiento en productos, la limpieza y las promociones.

Se agrupa entonces, en cuatro factores los elementos que tiene en cuenta el consumidor cuando va a hacer sus compras en los supermercados: características físicas del supermercado, variedad, calidad y economía y comodidad.

\section{Conclusiones}

Las diferentes variables que se midieron permitieron conocer en profundidad los elementos que el consumidor tiene en cuenta cuando va a comprar en la tienda y en el supermercado. Teniendo en cuenta la alta dimensionalidad de la medición, se realizó un agrupamiento en factores con las variables definidas, encontrando los siguientes para la tienda:

1. Con las relaciones que se pueden establecer durante la compra; (Factor externo - características del entorno: cultural social).

2. Con la forma como se entrega el servicio (factor interno: psicológico) y el producto en la tienda, (factor externo: características del establecimiento).

3. El valor agregado relacionado con horarios, economía y servicio a domicilio (factor externo: características del establecimiento).

4. La disponibilidad asociado con variedad de productos y la posibilidad de encontrar de todo, el hacer compras de urgencia y calidad de los productos. (Factor externo - característica del entorno: económico).

REDMARKA UIMA-Universidad de A Coruña - CIECID 
La disponibilidad también se encontró como variable relevante en el estudio de Goldman e Hino (2005) realizado en Israel. Las relaciones que se pueden establecer en la compra se evidenció en el estudio de Diaz, Lacayo y Salcedo (2007) que muestra resultados sobre América Latina, en el estudio de Ramirez (2008) en Barranquilla y Neiva y Baquero (2009) en Bogotá. Se observa entonces que la disponibilidad para obtener el servicio, son variables presentes en países como Israel, cobrando mayor importancia en América Latina y en Colombia las relaciones que se pueden establecer durante la compra, aspecto que se asocia con la cultura en estos países.

Para el caso de los supermercados se obtuvieron cuatro factores que agrupan:

1. Características físicas del supermercado (factor externo - características del establecimiento-distribución comercial).

2. La variedad en productos y marcas (factor externo - características del establecimiento - productos y servicios).

3. La calidad y economía en los productos y los servicios que ofrecen (factor externo - características del establecimiento y el entorno).

4. Comodidad (factor interno - psicológico).

El factor 3 sobre calidad y economía en los productos fue identificado también en el estudio de Farhangmehr et al., (2000) en Portugal. Mientras que en Tailandia (Gorton et al., 2011), la ventaja competitiva del supermercado se encuentra en la seguridad alimentaria, en la presente investigación no se identificó preocupación por este elemento, en el momento de decidir en donde comprar.

Se evidencia entonces, diferencias frente a los factores que influyen en la elección del consumidor frente a las tiendas y supermercados, observando que los factores externos (económicos y del establecimiento) están presentes en ambos casos, no

REDMARKA UIMA-Universidad de A Coruña - CIECID 
así para los factores del individuo y el componente cultural social que se identifica más en la elección de la tienda.

El componente cultural social, se identificó con mayor presencia en la elección de la tienda, lo cual se pudo comprender mejor a partir de los resultados en la fase cualitativa en donde se profundizó por ejemplo en las relaciones sociales y de confianza que se tejen en este establecimiento, las cuales son evidenciadas por la mayor disposición al diálogo con el tendero, compartiendo con éste, aspectos de la vida privada, o simplemente compartiendo temas de actualidad o posiciones ideológicas. El tendero en muchas ocasiones conoce la vida, los problemas sociales, económicos y hasta se convierte en el mejor amigo o hasta psicólogo de los usuarios.

Para este componente, se resalta también como resultado las características identificadas en el tendero las cuales se pueden asociar a la importancia que tiene en la elección de la tienda, el rol que representa entre sus clientes. De acuerdo con el análisis cualitativo el tendero cuenta con una buen autoimagen tanto en su capacidad para gestionar la tienda, como en sus características para atender al cliente (es un líder en su comunidad, está informado y se siente en capacidad de discutir diversos temas y en dar consejos a sus clientes).

\section{Bibliografía}

Baquero, M. (2009). La tienda de líchigo, una metáfora del consumo, Tesis de Maestría en Comunicación, Pontificia Universidad Javeriana, Bogota, Colombia.

REDMARKA UIMA-Universidad de A Coruña - CIECID 
Córdoba, L. J. y J.L. Cano. (2009). Estudio de hábitos y valores de los consumidores del canal tradicional tiendas de barrio de la ciudad de Cali, Entramado, 5(2), 44-63.

Departamento Administrativo Nacional de Estadística (DANE). Estratificación Socioeconómica, $\quad$ http://www.dane.gov.co/index.php/estratificacionsocioeconomica/generalidades, visitada el 19 de enero de 2016.

Díaz, A., Lacayo, J y L. Salcedo. (2007). Cómo vender a las tiendas de barrio en América Latina, The McKinsey Quarterly, 81-93.

Farhangmehr, M., Marques, S y J. Silva. (2000). Consumer and retailer perceptions of hypermarkets and traditional retail stores in Portugal, Journal of Retailing and Consumer Services, 7(4), 197-206.

Figuié, M y P. Moustier. (2009). Market appeal in an emerging economy: Supermarkets and poor consumers in Vietnam, Food Policy, 34(2), 210217.

Gary, M y C. Peter. (2011). Supermarket consumers and gender differences relating to their perceived importance levels of store characteristics, Journal of Retailing and Consumer Services, 18(6), 575-585.

Goldman, A y H. Hino. (2005). Supermarkets vs. traditional retail stores: diagnosing the barriers to supermarkets' market share growth in an ethnic minority community, Journal of Retailing and Consumer Services,12(4), 273-284.

REDMARKA UIMA-Universidad de A Coruña - CIECID 
Gorton, M., Sauer, J y P. Supatpongkul. (2011). Wet Markets, Supermarkets and the "Big Middle" for Food Retailing in Developing Countries: Evidence from Thailand, World Development, 39(9), 1624-1637.

Hair, J., Anderson, R., Tatham, R y W. Black. (1999). Análisis Multivariante. Madrid: Prentice Hall.

Hino, H. (2010). Antecedents of supermarket formats' adoption and usage: A study in the context of non-western customers, Journal of Retailing and Consumer Services, 17(1), 61-72.

Iglesias, I. (2014, 14 de Diciembre). El país no se satura aún de supermercados, Periódico El Tiempo, Bogotá http://www.eltiempo.com/economia/sectores/supermercados-encolombia/14924556, vistada el 29 de enero de 2016.

Linting, M., Meulman, J. J., Groenen, P. J y A.J. Van der Koojj. (2007). Nonlinear principal components analysis: Introduction and application, Psychological Methods, 12(3), 336-358.

Molina, M. O y D.L. Espinosa. (2010). Rotación en análisis de componentes principales categórico: Un caso práctico, Metodología de encuestas, 12, 6388.

Oficina Comercial de la Embajada de España en Colombia. (2007). Comercio y distribución en Colombia, Económico, Bogotá, pp. 4.

REDMARKA UIMA-Universidad de A Coruña - CIECID 
Páramo, M. D y P.E. Ramírez. (2009). Representaciones mentales de tenderos y consumidores de la tienda tradicional de barrio, Entornos, (22), 75-84.

Ramírez, P. E. (2008). ¿Por qué las tiendas de barrio en Colombia no han fracasado frente a la llegada de los supermercados?, Entornos, 1(21), 37 50.

Tiendas de barrio tienen el 53 por ciento del comercio minorista. (2014, 13 de Octubre). El Tiempo, Bogotá http://www.eltiempo.com/economia/sectores/aumentan-compras-en-tiendasde-barrio/14681117, visitada el 20 de febrero de 2016. 